\title{
Second-order distributed feedback lasers based on films containing perylenediimide derivatives
}

\author{
V. Navarro-Fuster*a,b ${ }^{*}$ P.G. Boj ${ }^{\text {a,c }}$, J.M. Villalvilla ${ }^{\mathrm{a}, \mathrm{b}}$, \\ J.A. Quintana a,c, M.A. Díaz-García ${ }^{\mathrm{a}, \mathrm{b}}$ \\ ${ }^{a}$ Instituto Universitario de Materiales de Alicante and Unidad Asociada UA-CSIC; ${ }^{\mathrm{b}}$ Dpto. Física \\ Aplicada, ${ }^{c}$ Dpto. Óptica, Universidad de Alicante, Alicante, 03080, Spain \\ V. Trabadelo ${ }^{\mathrm{d}, \mathrm{e}}$, A. Juarros ${ }^{\mathrm{e}}$, A. Retolaza, , S. Merino ${ }^{\mathrm{e}}$ \\ ${ }^{\mathrm{d}}$ Laboratory for Advanced Materials Processing, EMPA-Swiss Federal Laboratories for Materials \\ Testing and Research, Thun, CH-3602, Switzerland; ${ }^{\mathrm{e}}$ Micro and Nanotechnology Department; \\ Tekniker, Eibar, 20600, Spain
}

\begin{abstract}
The fabrication and characterization of highly efficient and photostable second-order distributed feedback lasers (DFB), based on polystyrene films doped with a perylenediimide (PDI) derivative as active laser material, is reported. DFB gratings were obtained via thermal nanoimprint lithography and transferred to $\mathrm{SiO}_{2}$ substrates by reactive ion etching. The influence of the PDI concentration on the laser performance has been investigated. The best results were obtained for the device based on the $0.5 \mathrm{wt} \%$ PDI-doped film, that emits at a wavelength of $571 \mathrm{~nm}$, with a threshold of $2.5 \mu \mathrm{J} /$ pulse and a photostability halflife of around $500 \mathrm{~min}$; i.e. 300000 pump pulses. The effectiveness of the DFB grating is evidenced by the decrease in threshold in two orders of magnitude with respect to the amplified spontaneous emission threshold obtained for a similar film deposited over a $\mathrm{SiO}_{2}$ substrate without grating.
\end{abstract}

Keywords: Distributed feedback laser, organics, perylenediimide, nanoimprint lithography

\section{INTRODUCTION}

Since the discovery of stimulated emission in semiconducting polymer films [1-3], extensive research has been devoted to the development of solid-state lasers based on semiconducting materials, including polymers, small organic molecules, oligomers, and dendrimers [4-6]. One of the major advantages of organic soluble materials is their easy processability in the form of thin films, by inexpensive techniques such as spin-coating, printing, etc. In addition, their wavelength can be tuned over a wide range, due to their broad photoluminescence (PL) spectrum [7]. One of the aspects that has received more attention in the literature, consists in achieving laser thresholds low enough to allow pumping with compact and inexpensive optical sources [8,9]. Another issue of great relevance in view of their possible commercialisation, that on the other hand has been less studied, is their photostability.

In the last years, the laser properties of films of a wide variety of organic materials have been reported [6,10-12]. Among them, polystyrene (PS) films doped with perylenediimide derivatives (PDIs) have demonstrated a great potential for the fabrication of lasers [13-15]. PDIs substituted at the imide nitrogen positions have shown very photostable amplified spontaneous emission (ASE) by optical pump, at low thresholds, when doped at low concentrations $(0.5-1$ wt\%) into PS $[13,14]$. These materials are particularly interesting in the field of data communications based on polymer optical fibres because they emit at wavelengths close to $570 \mathrm{~nm}$, which constitutes the second low-loss transmission window in poly(methylmethacrylate).

*victor.navarro@ua.es

Organic Photonics IV, edited by Paul L. Heremans, Reinder Coehoorn, Chihaya Adachi, Proc. of SPIE Vol. 7722, 77221G · (c) 2010 SPIE · CCC code: 0277-786X/10/\$18 - doi: 10.1117/12.854281 
One of the simplest laser resonators consists in placing the active medium between two mirrors, which is the method typically used with dyes [6]. Other configurations include vertical microcavities [3,4,6], whose thresholds are generally large, microrings, and microdisks $[4,6]$. Although the two latter devices generally show low thresholds, their interest for applications is limited by the fact that they do not provide a well-collimated beam of light. An alternative consists of using a distributed feedback (DFB) cavity in which a periodic modulation of the refractive index by a surface grating (on the substrate or on the active medium) is made in order to obtain light reflected by "Bragg-scattering" [10,16,17]. In DFB lasers, light propagating in a waveguide mode is scattered by the periodic corrugation, so the scattered light from each corrugation combines coherently and a new Bragg-scattered wave is formed, propagating in a new direction. Lasing occurs close to the so-called Bragg wavelength $\left(\lambda_{\text {Bragg }}\right)$ given by

$$
m \cdot \lambda_{\text {Bragg }}=2 \cdot n_{\text {eff }} \cdot \Lambda
$$

where $n_{\text {eff }}$ is the effective refractive index of the waveguide, $\Lambda$ is the period of the grating, and $m$ is the order of diffraction. For first-order diffraction $(m=1)$, light is diffracted out to the edge of the film, while for the case of secondorder diffraction $(m=2)$, light is diffracted out near perpendicular to the plane of the waveguide [18]. DFB structures present several advantages, such as single-mode emission (important for a number of applications), easy deposition of the organic film, low thresholds, and no need of mirrors. Numerous examples of optically pumped DFB organic lasers have been published in the literature $[4,6,11,18]$.

Various methods for grating recording, i.e., holography, electron-beam lithography, and soft lithography have been used. Among them, nanoimprint lithography (NIL) is one of the most promising technologies due to its high throughput, low-cost and high fidelity pattern transfer. Thermal NIL is based on the spatial displacement of a thermoplastic film under the concomitant application of pressure and temperature. A rigid nanostructured template is pressed onto the film and heated above its glass transition temperature $\left(T_{g}\right)$. Upon heating, the polymer becomes viscous and fills the cavities of the stamp, forming the pattern. Then, the system is cooled below $T_{g}$ and the stamp is demolded. NIL has already demonstrated its capacity to fabricate organic photonic devices, including DFB gratings $[6,19,20]$ and 2D photonic crystal lasers [21].

In this paper, we report on highly photostable solid-state lasers consisting of organic active layers deposited over DFB gratings fabricated by thermal NIL and subsequent transfer to $\mathrm{SiO}_{2}$ by dry etching methods. PS doped with the PDI derivative N,N'-di-(1-hexylheptyl) perylene-3,4:9,10-tetracarboxylic diimide (see chemical structure in Fig. 1) has been used as the organic gain medium and its concentration influence on the device performance is described.

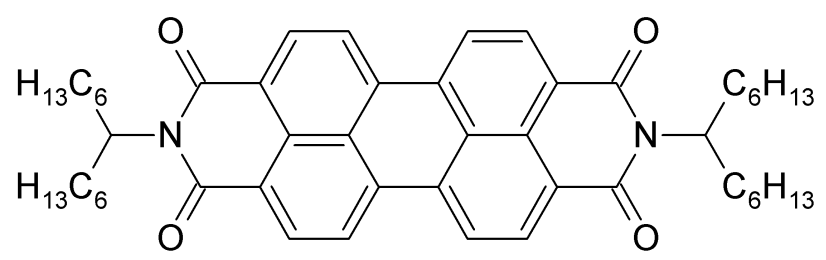

Fig. 1. Chemical structure of N,N'-di-(1-hexylheptyl) perylene-3,4:9,10-tetracarboxylic diimide. 


\section{EXPERIMENTAL SECTION}

\subsection{Waveguide modeling}

The samples used in this study constitute asymmetric planar waveguides since the refractive index of the polymer film $\left(n_{\mathrm{f}}\right)$ is larger than that of the $\mathrm{SiO}_{2}$ substrate $\left(n_{\mathrm{s}}\right)$ and the cover (air, $n_{\mathrm{c}}$ ). Particularly, at $\lambda=579 \mathrm{~nm}, n_{\mathrm{f}}=1.59, n_{\mathrm{s}}=$ 1.46 and $n_{c}=1$. In order to calculate the $n_{\text {eff }}$ values of the films, for a given film thickness and a certain wavelength, and thus estimate from Eq. 1, the appropriate grating period $\Lambda$ to have DFB emission at wavelengths within the PL emission spectrum of PDI, we have considered the propagation wave equation for the propagating modes of a waveguide without grating [22]:

$$
2 \pi \frac{h}{\lambda} \cdot \sqrt{n_{f}^{2}-n_{e f f}^{2}}-\phi_{c}-\phi_{s}=w \pi
$$

where $w$ is an integer that accounts for the mode order, $h$ is the thickness of the organic film and $\phi_{s}$ and $\phi_{c}$ are half of the phase changes at the interfaces between film and substrate and between film and air, given by

$$
\phi_{s}=\operatorname{arctg} \sqrt{\left(\frac{n_{f}}{n_{s}}\right)^{2 \rho} \frac{n_{e f f}^{2}-n_{s}^{2}}{n_{f}^{2}-n_{e f f}^{2}}} \quad \text { and } \quad \phi_{c}=\operatorname{arctg} \sqrt{\left(\frac{n_{f}}{n_{c}}\right)^{2 \rho} \frac{n_{e f f}^{2}-n_{c}^{2}}{n_{f}^{2}-n_{e f f}^{2}}}
$$

In these equations $\rho=0$ for TE modes and $\rho=1$ for TM modes.

Although the so-calculated $n_{\text {eff }}$ values for the waveguides without gratings differ from the real ones of the DFB sample, they are a good approximation to design the device.

\subsection{Fabrication of relief gratings over $\mathrm{SiO}_{2}$ substrates}

A silicon master grating with a size of $(2 \times 2) \mathrm{mm}^{2}$, period $\Lambda=368 \mathrm{~nm}$ and equal line and space was first fabricated by e-beam lithography (Kelvin Nanotechnology Ltd.). This period was selected in order to get a $\lambda_{\text {Bragg }}$ value of $579 \mathrm{~nm}$, which is close to the vibrational PL peak of PDI-doped films, at which ASE generally takes place. This value satisfies eq. (1) for $m=2$ and $n_{\text {eff }} \sim 1.57$ (for a $1 \mu \mathrm{m}$ thick film of $0.5 \mathrm{wt}$. \% PDI-doped PS film). The stamp was subsequently treated with a fluorosilane-based antiadhesive coating deposited from the vapor phase [23], in order to avoid adhesion of the polymer to the stamp during the imprinting process and to facilitate demolding. The stamp was imprinted onto a mrI8030E (from Micro Resist Technology $\mathrm{GmbH}$ ) thermoplastic polymer film spin-coated on a thermally oxidized 4 in. silicon wafer. The embossing was carried out in a Jenoptik HEX03 press under vacuum at $180{ }^{\circ} \mathrm{C}$ and the applied force $\left(20000 \mathrm{~N}\right.$ ) was held for $900 \mathrm{~s}$. The demolding temperature was $60^{\circ} \mathrm{C}$. The residual layer was removed under an $\mathrm{O}_{2}$ plasma. The grating was transferred to the $\mathrm{SiO}_{2}$ substrate by CHF3/Ar plasma etching in an Oxford PlasmaLab ICP-80. The final grating over $\mathrm{SiO}_{2}$ had a period of $368 \mathrm{~nm}$ and a depth of $220 \mathrm{~nm}$. 


\subsection{Deposition of organic active films}

Toluene solutions containing PS as inert polymer and varying concentrations of PDI, ranging from 0.09 to $1 \mathrm{wt}$ \%, were spin-coated at $3000 \mathrm{rpm}$ over $\mathrm{SiO}_{2}$ gratings. The percentage of PS with respect to the solvent was adjusted in order to get films with thickness below $600 \mathrm{~nm}$. According to eqs. 2 and 3, such films would propagate at $\lambda=579 \mathrm{~nm}$ one mode (one TE and one TM). In a previous work [15], we fabricated devices based on $1 \mu \mathrm{m}$-thick films that showed two DFB peaks, that were attributed to two of the propagating modes of the waveguide. Thus, the preparation of thinner films in this work is justified by our aim to obtain single-mode emission. Final thickness, measured in a film without grating with an interferometer coupled to a microscope, was $590 \mathrm{~nm}$.

To properly assess the effect of the grating in the emission characteristics, we also deposited films of similar characteristics over substrates without gratings and characterized their emission properties. These samples were used to obtain the absorption spectra, by means of a Jasco V650 spectrophotometer, and to determine film thickness, with an interferometer coupled to an optical microscope. Standard photoluminescence (PL) spectra were obtained in a Jasco FP6500 fluorimeter by exciting the samples at $490 \mathrm{~nm}$ and then collecting the transmitted beam at $45^{\circ}$. The excitation wavelength was $490 \mathrm{~nm}$, instead of the $532 \mathrm{~nm}$ beam used in the DFB characterization below, in order to reduce its overlap with the PL emission.

\subsection{DFB characterization}

For the characterization of the emission properties of the DFB lasers, as well as of the samples without gratings, a pulsed Nd:YAG laser $(10 \mathrm{~ns}, 10 \mathrm{~Hz})$ operating at $532 \mathrm{~nm}$ was used. This excitation wavelength lies in the absorption region of the PDI-doped films (see Fig. 2). The energy of the pulses was controlled using neutral density filters. The pump laser beam was expanded and collimated and only the central part was used in order to ensure uniform intensity. The pump beam was incident upon the samples at $20^{\circ}$ with respect to the normal of the sample plane. The spot size of the beam on the film was $0.01 \mathrm{~cm}^{2}$. The emitted laser light was collected perpendicular to the surface with a fiber spectrometer placed at $1 \mathrm{~cm}$ from the sample.

\section{RESULTS AND DISCUSSION}

The concentration dependence of the DFB emission has been studied in terms of threshold, emission wavelength and linewidth. PS films containing different concentrations (0.09, 0.3, 0.5 and 1 wt. \%) of PDI were deposited over $\mathrm{SiO}_{2}$ substrates with gratings, as well as over $\mathrm{SiO}_{2}$ without gratings. Fig. 2 shows the absorption and PL spectra for the 0.5 wt. $\%$ PDI-doped film deposited over $\mathrm{SiO}_{2}$ without grating. Note that due to the small area of the DFB device, their corresponding spectra could not be obtained. As observed in Fig. 2, the absorption spectrum shows a peak at $527 \mathrm{~nm}$, attributed to the perylene core $\pi-\pi^{*}$ transition. A characteristic vibronic fine structure in the high-energy side is observed. The PL spectrum shows a main peak PL(0-0) at $535 \mathrm{~nm}$ and a vibrational transition PL(0-1) at $576 \mathrm{~nm}$. In the range of concentrations studied, no changes in the shape of the spectra were observed. However, it should be noted that in previous works [13] changes in the PL spectrum for concentrations larger than $2 \mathrm{wt}$ \%, attributed to the formation of aggregates species, were reported. 


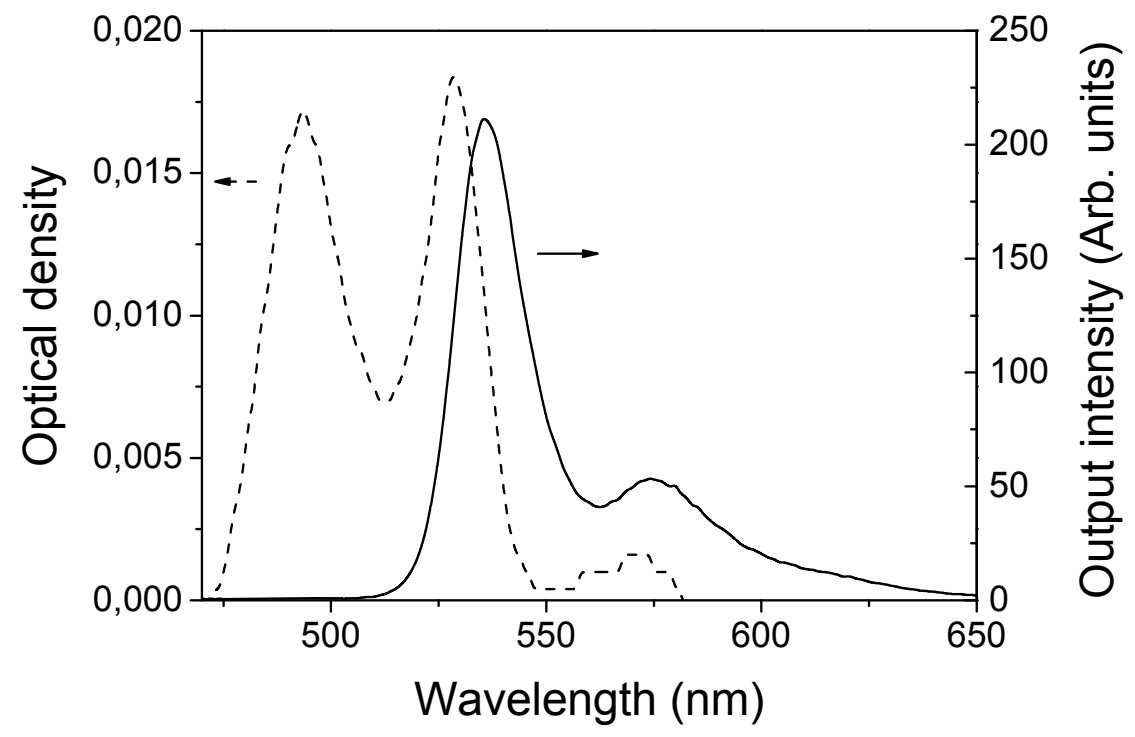

Fig. 2. Absorption (dashed curve, left axis) and PL spectra (full curve, right axis) of a 0.5 wt. \% PDI-doped PS film.

For all the devices prepared, laser emission was observed at around $571 \mathrm{~nm}$, with a linewidth (defined as the full width at half-maximum, FWHM) of less than $2 \mathrm{~nm}$ (limited by the resolution of the spectrophotometer). No differences in terms of linewidth and wavelength could be appreciated for the range of PDI concentrations studied. Fig. 3 shows the emission spectra at low and high pump intensities for the DFB device based on a 0.5 wt.\% PDI-doped film (Fig. 3a), as well as for a sample consisting of a similar film but deposited over $\mathrm{SiO}_{2}$ without grating (Fig. 3b). In this latter case, no real lasing takes place since no cavity is present. Nevertheless, gain narrowing at a certain pump intensity, due to ASE is observed, as already discussed in detail in previous works [13-14]. By comparing Figs. 3a and 3b, it is clear that the presence of the grating leads to changes in the shape of the emission spectra. At low pump intensity, the output spectrum of the DFB device is broad and rather different than the one obtained with the sample without a grating. In accordance with other works $[10,18]$, the modifications observed by the presence of the grating are attributed to waveguided PL that has been Bragg scattered out of the waveguide at an angle normal to the substrate. It is also observed that the spectrum below threshold of the DFB device exhibits a characteristic dip in the emission intensity at around $571 \mathrm{~nm}$, due to an inhibition of the propagation of the waveguide light by the grating. This Bragg dip can be envisaged as a photonic stop band for waveguided modes. The spectrum above threshold shows a narrow and intense peak close to the Bragg dip.

In the case of the film deposited over $\mathrm{SiO}_{2}$ without grating (Fig. 3b), the spectrum obtained at low pump intensity does not show a dip similar to that observed in Fig. 3a for the sample with grating. With respect to the high pump intensity spectrum, due to ASE, its linewidth is clearly reduced (up to around $4 \mathrm{~nm}$ ) with respect to the low pump intensity one and its total output intensity (at $578 \mathrm{~nm}$ ) is drastically increased. Note that the obtained ASE linewidth is larger than that measured with the DFB structure $(<2 \mathrm{~nm})$. In addition, the emission wavelength also differs from that obtained in the DFB device. 

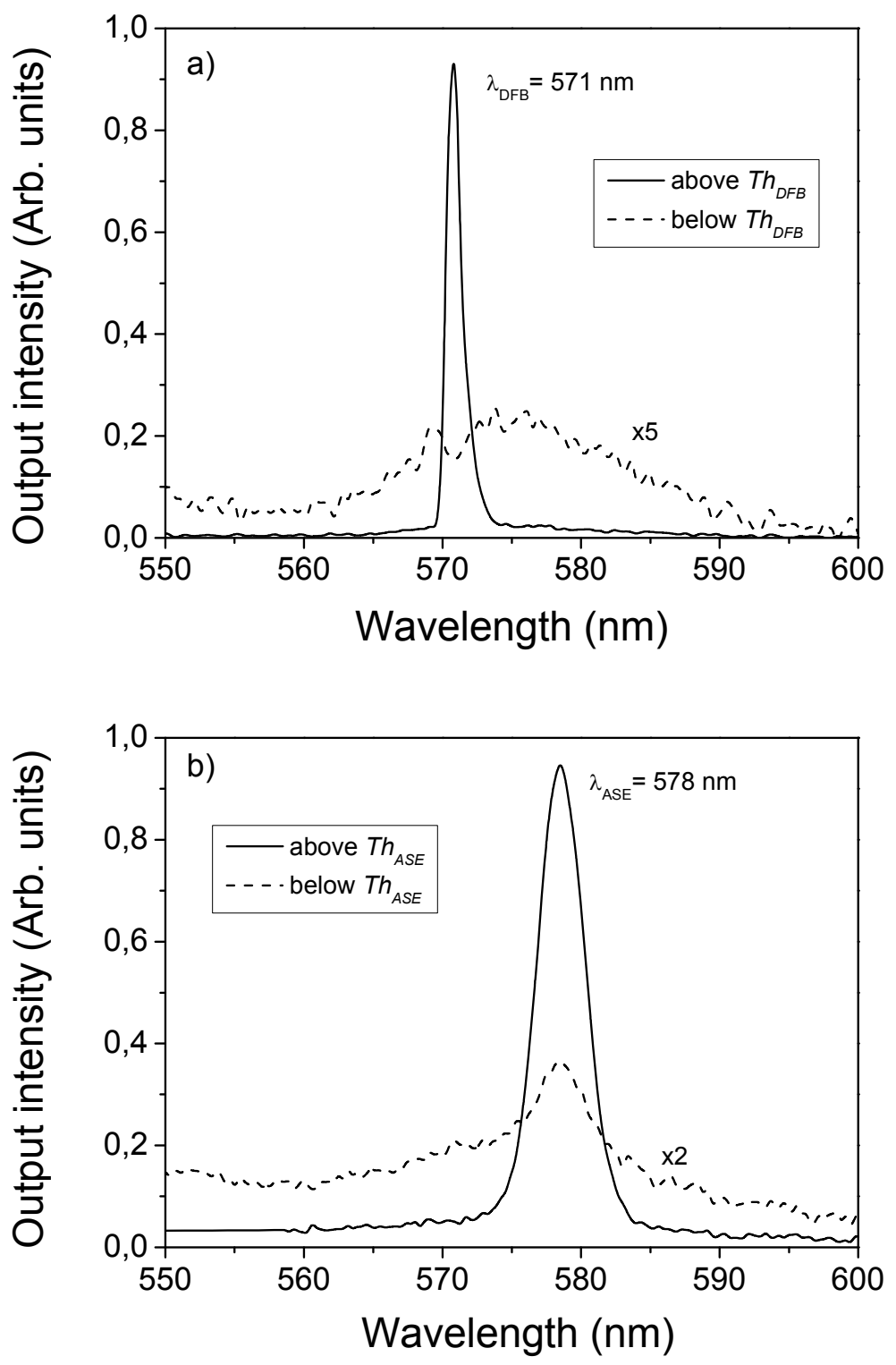

Fig. 3. Emission spectra of a 0.5 wt. \% PDI-doped PS film deposited over $\mathrm{SiO}_{2}$ with DFB grating (a) below the laser threshold $T h_{\mathrm{DFB}}$ intensity $(1.5 \mu \mathrm{J} /$ pulse, dashed curve $)$ and above $T h_{\mathrm{DFB}}(4.5 \mu \mathrm{J} /$ pulse, solid curve $)$ and without grating (b) below the ASE threshold $T h_{\mathrm{ASE}}$ intensity $(70 \mu \mathrm{J} /$ pulse, dashed curve $)$ and above $T h_{\mathrm{ASE}}(510 \mu \mathrm{J} / \mathrm{pulse}$, solid curve).

DFB thresholds were determined from the output intensity versus pump intensity curves (see Fig. 4). The best results were obtained for the $0.5 \mathrm{wt}$. \% PDI-doped film, with a threshold of $2.5 \mu \mathrm{J} /$ pulse. In order to evaluate the effect of the grating, results obtained for the samples without gratings have also been included. In this case no significant differences were observed among the various PDI concentrations, thus only data for the $0.5 \mathrm{wt} \%$-doped film have been included. As observed in Fig. 4, for the DFB lasers, a nearly exponential increase is clearly observed for pump intensities above the laser threshold. For the sample without grating, a change in slope is not clearly defined, thus the numerical value of the ASE threshold ( $125 \mu \mathrm{J} /$ pulse) was obtained by determining the intensity at which the FWHM of the emitted light decays to half of its maximum value. Note that the ASE threshold is around two orders of magnitude larger than that of the DFB laser, indicating the good efficiency of the grating. 


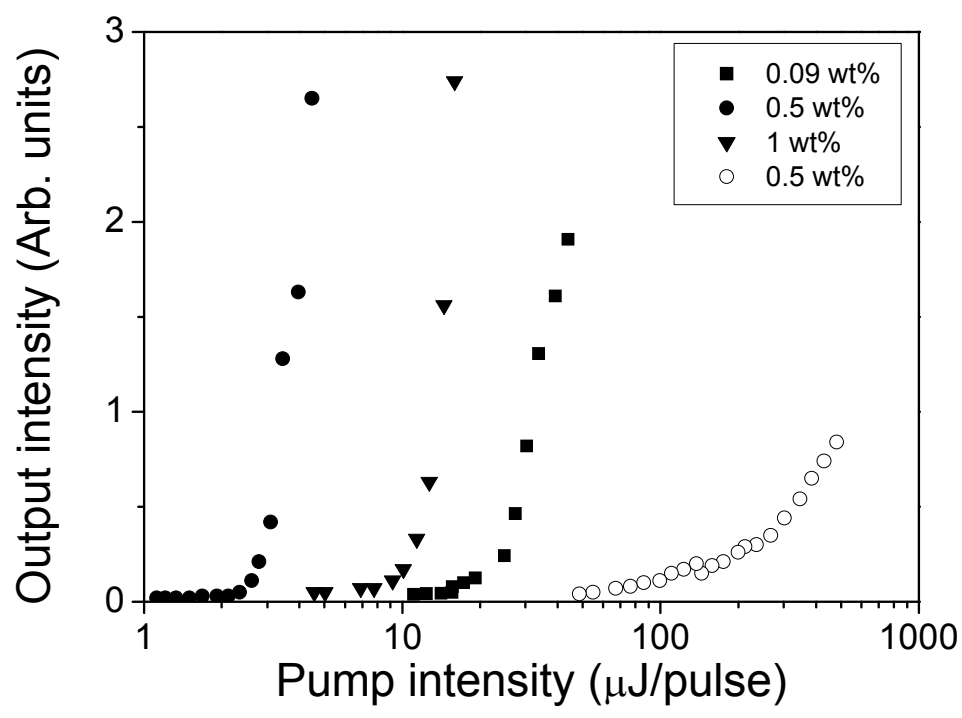

Fig. 4. Laser output intensity versus pump intensity for DFB lasers based on films doped with different PDI concentrations (full symbols). For comparison purposes the ASE output intensity for the $0.5 \mathrm{wt}$. \% PDI-doped film without grating has been included (open symbols).

The photostability of the devices was studied by recording the total DFB intensity emitted above the threshold, at constant pump intensity and over the same spot of the material, as a function of time. Results obtained for the $0.5 \mathrm{wt}$ \% PDI-doped film are displayed in Fig. 5. The number of pump pulses has also been included in the top margin of the figure, in order to be able to compare with other lasers, independently on the repetition rate of the excitation source.

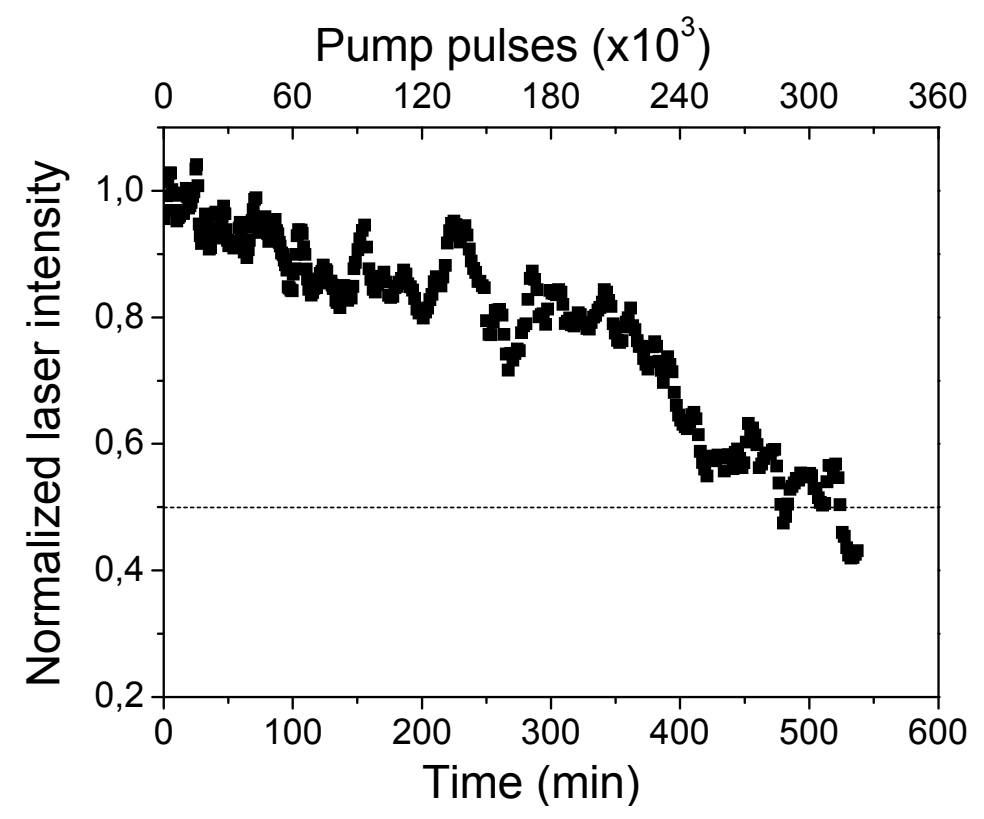

Fig. 5. Normalized laser intensity versus irradiation time (bottom axis) and versus the number of pump pulses (10 ns, $10 \mathrm{~Hz}$; top axis) for a DFB device based on a $0.5 \mathrm{wt} \%$ PDI-doped PS film, at a pump intensity of $4 \mu \mathrm{J} / \mathrm{pulse}$. 
A parameter often used to quantify the photostability of a device is the so-called photostability halflife $\left(\tau_{1 / 2}\right)$, i.e. the time or number of pump pulses at which the laser emission intensity decays to half of its maximum value. As observed in Fig. 5, for an excitation intensity just above the threshold, $\tau_{1 / 2}$ values of around 500 min (300000 pump pulses) have been obtained. These values are much larger than those reported previously [15] for the ASE photostability of other PDIdoped films deposited over substrates without gratings, were values around one order of magnitude larger were obtained. This substantial improvement in the photostability performance is mainly due to the decrease in threshold. This indicates that the advantage of reducing the laser threshold of the device is important, not only to allow using less powerful and more compact excitation sources, but also to improve the photostabilty, that is one of the most important parameters from a practical point of view.

\section{CONCLUSIONS}

Highly efficient and photostable second-order distributed feedback organic lasers, fabricated by thermal nanoimprint lithography and dry etching methods, have been fabricated and characterized. Polystyrene films doped with a perylenediimide (PDI) derivative have been used as active laser material. The influence of the PDI content in the laser performance has been investigated. The most efficient device was the one based on a $0.5 \mathrm{wt}$. \% PDI-doped film, that emit at wavelength of $571 \mathrm{~nm}$, with threshold of $2.5 \mu \mathrm{J} /$ pulse and a photostabilility halflife of around 300000 pump pulses.

\section{ACKNOWLEDGMENT}

The authors acknowledge support from the Spanish Government MEC and the European Community (FEDER) through grant number MAT2008-06648-C02. We also thank V. Esteve for technical assistance.

\section{REFERENCES}

1. Hide, F., Schwartz, B. J., Díaz-García, M. A., and Heeger, A. J., "Laser emission from solutions and films containing semiconducting polymers and titanium dioxide nanocrystals," Chem. Phys. Lett. 256, 424-430 (1996).

2. Hide, F., Diaz-Garcia, M. A., Schwartz, B. J., Andersson, M.,Q. Pei and Heeger, A. J., "Semiconducting polymers: a new class of solid-state laser materials," Science 273, 1833-1836 (1996).

3. Tessler, N., Denton, G. J. and Friend, R. H., "Lasing from conjugated-polymer microcavities," Nature 382, 695-697 (1996).

4. McGehee, M. D. and Heeger, A. J., "Semiconducting (conjugated) polymers as materials for solid-state lasers," Adv. Mater. 12, 1655-1668 (2000) and references therein.

5. Tessler, N., "Lasers based on semiconducting organic materials," Adv. Mater. 11, 363-370 (1999) and references therein.

6. Samuel, I. D. W. and Turnbull, G. A., “Organic semiconductor lasers,” Chem. Rev. 107, 1272-1295 (2007) and references therein.

7. Duarte, F. J. and Hillman, L.W., eds., [Dye Laser Principles with Applications], Academic (1990).

8. Turnbull, G. A., Andrew, P., Barnes, W. L. and Samuel, I. D. W., "Operating characteristic of a semiconducting polymer laser pumped by a microchip laser,” Appl. Phys. Lett. 82, 313-315 (2003).

9. Vasdekis, A. E., Moore, S. A., Ruseckas, A., Krauss, T. F., Samuel, I. D. W. and Turnbull, G. A., "Silicon based organic semiconductor laser," Appl. Phys. Lett. 91, 051124 (2007).

10. Calzado, E.M., Villavilla, Boj, P.G., Quintana, J.A., Postigo, P.A. and Díaz-García, M.A., "Blue surface-emitting distributed feedback laser based on TPD-doped films," Appl. Opt. 49, 463-470 (2010).

11. Calzado, E. M., Villalvilla, J. M., Boj, P. G., Quintana, J. A. and Díaz-García, M. A., "Concentration dependence of amplified spontaneous emission in organic-based waveguides,” Org. Electron. 7, 319 (2006). 
12. Díaz-García, M.A., Calzado, E.M., Villalvilla, J.M., Boj, P.G., Quintana, J. A., Giacalone, F., Segura, J. L. and Martín, N., "Concentration dependence of amplified spontaneous emission in two oligo-(p-phenylenevinylene)," J. Appl. Phys. 97, 063522 (2005).

13. Calzado, E. M., Villalvilla, J. M., Boj, P. G., Quintana, J. A., Gómez, R., Segura, J. L. and Díaz-García, M. A., "Amplified spontaneous emission in polymer films doped with a perylenediimide derivative," Appl. Opt. 46, 38363842 (2007).

14. Calzado, E. M., Villalvilla, J. M., Boj, P. G., Quintana, J. A., Gómez, R., Segura, J. L. and Díaz-García, M. A., "Effect of structural modifications in the spectral and laser properties of perylenediimide derivatives," J. Phys. Chem. C 111, 13595 (2007).

15. Trabadelo, V., Juarros, A., Retolaza, A., Merino, S., Ramírez, M.G., Navarro-Fuster, V., Villalvilla, J.M., Boj, P.G., Quintana, J.A., Díaz-García, M.A., "Highly photostable solid-state organic distributed feedback laser fabricated via thermal nanoimprint lithography," Microelectronic Engineering 87, 1428-1430 (2010).

16. Coldren, L. A. and Corzine, S. W., [Diode Lasers and Photonic Integrated Circuits] Wiley (1995).

17. McGehee, M. D., Díaz-García, M. A., Hide, F., Gupta, R., Miller, E. K., Moses, D. and Heeger, A. J., "Semiconducting polymer distributed feedback laser," Appl. Phys. Lett. 72, 1536-1538 (1998).

18. Turnbull, G. A., Andrew, P., Jory, M. J., Barnes, W. L. and Samuel, I. D.W., "Relationship between photonic band structure and emission characteristic of a polymer distributed feedback laser," Phys. Rev. B 64, 125122 (2001).

19. Chou, S.Y., Krauss, P.R. and Renstrom, P.J., "Imprint Lithography with 25-Nanometer Resolution", Science, 272, 85-87 (1996).

20. Christiansen, M.B., Shøler, M., Kristensen, A., "Integration of active and passive polymer optics", Opt. Exp. 15, 3931-3939 (2007).

21. Reboud, V., Lovera, P., Kehagias, N., Zelsmann, M., Schuster, C., Reuther, F., Gruetzner, G., Redmond, G. and Sotomayor Torres, C. M., "Two-dimensional polymer photonic crystal band-edge lasers fabricated by nanoimprint lithography," Appl. Phys. Lett. 91, 151101 (2007).

22. Marcuse, D., [Theory of dielectric optical waveguides], second edition, Academic Pr., 1-19 (1991).

23. Schift, H, Saxer, S, Park, S., Padeste, C., Pieles, U. and Gobrecht, J., "Controlled co-evaporation of silanes for nanoimprint stamps," Nanotechnology 16, S171-S175 (2005). 